\title{
THE MULTIPLE REFERENCE PRINCIPLE COMPONENT LEAST MEAN SQUARES ALGORITHM: A PROJECTION BASED APPROACH
}

\author{
G. Nijsse, J. Van Dijk and J.B. Jonker \\ University of Twente, Fac. of Engineering Technology, Dep. of Mechanical Engineering, \\ Lab. of Mechanical Automation, P.O. Box 217, 7500 AE Enschede, The Netherlands
}

\begin{abstract}
The principle component least mean squares algorithm (PCLMS) is an elegant adaptive control algorithm for cancelling a tonal disturbance signal in active control applications, such as active noise control and active vibration isolation control. The algorithm removes the spatial correlation between the actuator inputs and the error sensor outputs to enable fast convergence of the adaptive controller. However, a drawback of the PCLMS algorithm is that it can only suppress a disturbance signal which contains a single frequency component. The contribution of this paper is that we present a numerically robust projection based approach in which the PCLMS is extended with the ability to suppress a disturbance signal which contains multiple frequency components. The potential of the algorithm is demonstrated by multi tonal control on a realistic model of a real-time vibration isolation set-up. The algorithm is shown to outperform the traditional filtered-x least mean squares algorithm.
\end{abstract}

\section{INTRODUCTION}

A problem which is often encountered in active control applications, like active noise control and active vibration isolation control, is that a disturbance signal need to be suppressed which consists of multiple tonal components. For this purpose, the filtered-x least mean square (FxLMS) algorithm is commonly deployed. However, a drawback of the FxLMS algorithm is that the convergence speed is dependent on the spatial correlation (i.e. coupling) present in the transfer path between the actuator inputs and the error sensor outputs [1]. In an attempt to overcome this problem, the principle component least mean squares (PCLMS) algorithm was developed which removes the spatial correlation [2]. On contrast to the FxLMS algorithm however, the PCLMS algorithm can only suppress a disturbance signal which contains a single frequency component.

In this paper we solve this problem for a disturbance signal consisting of multiple frequency components. A numerically robust projection based approach is presented in which the PCLMS algorithm is extended with the ability to suppress a disturbance signal which contains $J>1$ frequency components. The approach is based on creating $J$ decoupled filtered error sensor signals. Every filtered error sensor signal contains the contribution of a single frequency component only. Based on the filtered error sensor signals, $J$ adaptive controllers can be updated separately with the PCLMS algorithm. The new algorithm is referred to as multiple reference PCLMS (MR-PCLMS)

This paper is organized as follows. Section 2 describes the traditional (single reference) PCLMS algorithm for clarity [2]. In section 3 the MRPCLMS algorithm is developed. The MR-PCLMS algorithm is validated in section 4 on a realistic model of a vibration isolation set-up. Finally, section 5 gives the conclusions of the paper.

\section{SINGLE REFERENCE PCLMS}

In figure 1 a block diagram of the adaptive control problem is depicted [1]. $\quad \mathbf{P}\left(q^{-1}\right)$ represents

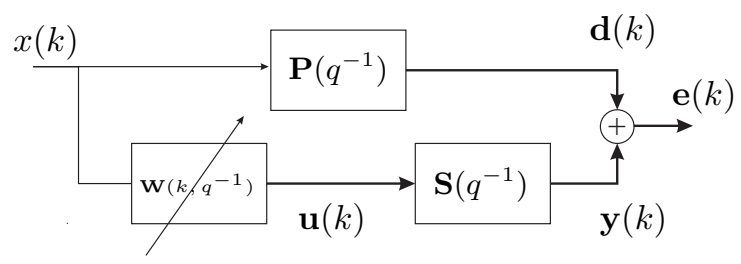

Fig. 1. Adaptive control problem.

the linear time invariant (LTI) $M \times 1$ transfer path from the reference input $x(k)$ to the error sensor output $\mathbf{e}(k)$ i.e. the primary path (i.e. $M$ is the number of error sensors). $q^{-1}$ is the backward 
shift operator and ' $\mathrm{k}$ ' is the sample number normalized with the sample time $T . x(k)$ contains a single frequency component of normalized frequency $2 \pi f_{1} T=\omega_{1} T$ ( $f_{1}$ represents the disturbance frequency in $\mathrm{Hz}) . \mathbf{S}\left(q^{-1}\right)$ represents the LTI $M \times K$ transfer path from $\mathbf{u}(k)$ to $\mathbf{e}(k)$ i.e. the secondary path (i.e. $K$ is the number of actuators). $\mathbf{W}\left(k, q^{-1}\right)$ is the $K \times 1$ adaptive finite impulse response (FIR) controller which denotes the transfer path from $x(k)$ to $\mathbf{u}(k)$ :

$$
\mathbf{W}\left(k, q^{-1}\right)=\left[\begin{array}{c}
w_{1}^{(1)}(k)+w_{1}^{(2)}(k) q^{-1} \\
\vdots \\
w_{K}^{(1)}(k)+w_{K}^{(2)}(k) q^{-1}
\end{array}\right],
$$

The goal of the adaptive controller $\mathbf{W}\left(k, q^{-1}\right)$ is to minimize $\mathbf{e}(k)$. This is done by counteracting the disturbance signal $\mathbf{d}(k)$ with the anti-disturbance signal $\mathbf{y}(k)$, by adapting the controller parameters $w_{m}^{(l)}(k),(m=1, \ldots, K),(l=1,2)$, appropriately. Next, we derive the PCLMS algorithm.

Assume that $\mathbf{e}(k)$ has time to reach its steady state at each sample $k$ [1]. In the frequency domain, the complex error sensor signal $\widetilde{\mathbf{e}}(k)$ can be written in shorthand notation as [1,2]:

$$
\widetilde{\mathbf{e}}(k)=\widetilde{\mathbf{d}}+\widetilde{\mathbf{S}} \widetilde{\mathbf{W}}(k),
$$

where $\widetilde{\mathbf{d}} \in \mathbb{C}^{M \times 1}$ and $\widetilde{\mathbf{W}}(k) \in \mathbb{C}^{K \times 1}$ provide the amplitude and phase information of $\mathbf{d}(k)$ and $\mathbf{W}\left(k, q^{-1}\right)$ at normalized frequency $\omega_{1} T$, respectively (note that $\widetilde{\mathbf{d}}$ does not depend on ' $\mathrm{k}$ '). $\widetilde{\mathbf{S}} \in$ $\mathbb{C}^{M \times K}$ is the complex plant response of $\mathbf{S}\left(q^{-1}\right)$ at normalized frequency $\omega_{1} T$. If a singular value decomposition (SVD) of $\widetilde{\mathbf{S}}$ is computed, this gives $\widetilde{\mathbf{S}}=\mathbf{U} \boldsymbol{\Sigma} \mathbf{V}^{H}$, with $(\cdot)^{H}$ the Hermitian transpose, $\mathbf{U} \in \mathbb{C}^{M \times M}, \boldsymbol{\Sigma} \in \mathbb{R}^{M \times K}$ and $\mathbf{V} \in \mathbb{C}^{K \times K}$. The SVD solution can be substituted in equation (2):

$$
\widetilde{\mathbf{e}}(k)=\widetilde{\mathbf{d}}+\mathbf{U} \boldsymbol{\Sigma} \mathbf{V}^{H} \widetilde{\mathbf{W}}(k) .
$$

By pre-multiplying the left hand side and the right hand side in equation (3) with $\mathbf{U}^{H}$ and by making use of the fact that $\mathbf{U}^{H} \mathbf{U}=\mathbf{I}$, with $\mathbf{I} \in \mathbb{R}^{M \times M}$ the identity matrix, equation (3) can be written in shorthand as:

$$
\overline{\widetilde{\mathbf{e}}}(k)=\overline{\widetilde{\mathbf{d}}}+\boldsymbol{\Sigma} \overline{\widetilde{\mathbf{W}}}(k),
$$

with $\overline{\widetilde{\mathbf{e}}}(k)=\mathbf{U}^{H} \widetilde{\mathbf{e}}(k), \overline{\widetilde{\mathbf{d}}}=\mathbf{U}^{H} \widetilde{\mathbf{d}}$ and $\overline{\widetilde{\mathbf{W}}}(k)=$ $\mathbf{V}^{H} \widetilde{\mathbf{W}}(k)$. Equation (4) forms the basis for the PCLMS algorithm. Instead of determining the controller $\widetilde{\mathbf{W}}(k)$ as in equation (2), the PCLMS algorithm determines the controller $\overline{\widetilde{\mathbf{W}}}(k)$ as in equation (4). In figure 2 the PCLMS adaptive control problem is given. In the figure the argument $q^{-1}$ has been dropped from the filters. Furthermore, $\overline{\mathbf{W}}\left(k, q^{-1}\right)$ (or $\overline{\mathbf{W}}(k)$ without $q^{-1}$ ) is the time domain counterpart of $\widetilde{\mathbf{W}}(k)$. Note that in the implementation, every element of the matrices $\mathbf{U}^{H}$ and $\mathbf{V}$ needs to be transformed to, for example, a two-parameter FIR model to obtain the filters $\mathbf{U}^{H}\left(q^{-1}\right)$ and $\mathbf{V}\left(q^{-1}\right)$. Minimizing $J(k)=$

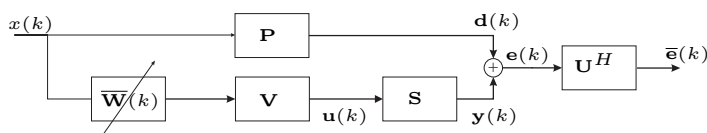

Fig. 2. PCLMS adaptive control problem.

$\overline{\mathbf{e}}^{T}(k) \overline{\mathbf{e}}(k)$ with respect to $\overline{\mathbf{w}}(k)$, and using the least mean squares filter update, gives [1]:

$$
\overline{\mathbf{w}}(k+1)=\overline{\mathbf{w}}(k)-\frac{\mu}{2} \frac{\partial \overline{\mathbf{e}}^{T}(k) \overline{\mathbf{e}}(k)}{\partial \overline{\mathbf{w}}(k)},
$$

which gives the PCLMS algorithm. Column vector $\overline{\mathbf{w}}(k) \in \mathbb{R}^{2 K}$ contains the controller parameters $\bar{w}_{m}^{(l)}(k),(m=1, \ldots, K),(l=1,2)$, of the controller $\overline{\mathbf{W}}\left(k, q^{-1}\right)$. The latter is defined equivalently as the one in equation (1) with $\bar{w}_{m}^{(l)}(k)$ substituted for $w_{m}^{(l)}(k) . \mu$ is the step-size which need to be chosen by the user $[2,1]$. Next we generalize the results to $J$ reference signals.

\section{MULTIPLE REFERENCE PCLMS}

Now assume $\mathbf{P}\left(q^{-1}\right)$ is a $M \times J$ LTI transfer path and that the reference signal is given by $\mathbf{x}(k)=$ $\left[x_{1}(k) x_{2}(k) \ldots x_{J}(k)\right]^{T} \in \mathbb{R}^{J}$. Every scalar reference signal $x_{l}(k),(l=1, \cdots, J)$, contains a single frequency component of normalized frequency $2 \pi f_{l} T=\omega_{l} T$. Assuming that $\mathbf{S}\left(q^{-1}\right)$ is LTI, $\widetilde{\mathbf{e}}(k)$ can be written as:

$$
\widetilde{\mathbf{e}}(k)=\widetilde{\mathbf{d}}+\widetilde{\mathbf{S}}_{1} \widetilde{\mathbf{W}}_{1}(k)+\ldots+\widetilde{\mathbf{S}}_{J} \widetilde{\mathbf{W}}_{J}(k),
$$

with $\widetilde{\mathbf{S}}_{l} \in \mathbb{C}^{M \times K},(l=1, \ldots, J)$, the complex plant response of $\mathbf{S}\left(q^{-1}\right)$ at normalized frequency $\omega_{l} T . \widetilde{\mathbf{W}}_{l}(k) \in \mathbb{C}^{K \times 1},(l=1, \ldots, J)$, provides the amplitude and phase information of $\mathbf{W}_{l}\left(k, q^{-1}\right)$, at normalized frequency $\omega_{1} T$. We now assume that $\widetilde{\mathbf{d}}\left(=\widetilde{\mathbf{P}}_{1}+\ldots+\widetilde{\mathbf{P}}_{J}\right)$ can be rewritten to $\widetilde{\mathbf{d}}=$ $\widetilde{\mathbf{S}}_{1} \widetilde{\mathbf{W}}_{1}^{o}+\ldots+\widetilde{\mathbf{S}}_{J} \widetilde{\mathbf{W}}_{J}^{o}$, with $\widetilde{\mathbf{P}}_{l} \in \mathbb{C}^{M \times 1},(l=$ $1, \ldots, J)$, the complex plant response of $\mathbf{P}\left(q^{-1}\right)$ at normalized frequency $\omega_{l} T$ from input $l$ to all $M$ outputs. With $\widetilde{\mathbf{W}}_{l}^{o} \in \mathbb{C}^{K \times 1},(l=1, \ldots, J)$, the optimal time invariant controller parameters. Now equation (5) can be rewritten to:

$$
\begin{aligned}
\widetilde{\mathbf{e}}(k)= & \widetilde{\mathbf{S}}_{1} \widetilde{\mathbf{W}}_{1}^{o}+\ldots+\widetilde{\mathbf{S}}_{J} \widetilde{\mathbf{W}}_{J}^{o}+\ldots \\
& \ldots+\widetilde{\mathbf{S}}_{1} \widetilde{\mathbf{W}}_{1}(k)+\ldots+\widetilde{\mathbf{S}}_{J} \widetilde{\mathbf{W}}_{J}(k) .
\end{aligned}
$$


We further rewrite equation (6) as:

$$
\widetilde{\mathbf{e}}(k)=\widetilde{\mathbf{S}}_{l}\left(\widetilde{\mathbf{W}}_{l}^{o}+\widetilde{\mathbf{W}}_{l}\right)+\mathcal{S}_{l} \mathcal{W}_{l},
$$

where $\mathcal{S}_{l} \in \mathbb{C}^{M \times K(J-1)}$ is defined as:

$$
\mathcal{S}_{l}=\left[\begin{array}{llllll}
\widetilde{\mathbf{S}}_{i} & \ldots & \widetilde{\mathbf{S}}_{m} & \widetilde{\mathbf{S}}_{n} & \ldots & \widetilde{\mathbf{S}}_{p}
\end{array}\right]
$$

and $\mathcal{W}_{l}(k)=$

$$
\left[\mathbb{W}_{i}(k) \ldots \mathbb{W}_{m}(k) \mathbb{W}_{n}(k) \ldots \mathbb{W}_{p}(k)\right]^{H}
$$

with $\mathbb{W}_{r}(k)=\left(\widetilde{\mathbf{W}}_{r}^{o}+\widetilde{\mathbf{W}}_{r}(k)\right)^{H}, r=i, m, n, p$. In equations (8) and (9) the indices are defined as follows. If $l=1: i=2, p=J, m=i+1$ and $n=m+1$. If $l=J: i=1, p=J-1, m=i+1$ and $n=m+1$. If $l=2, \ldots, J-1: i=1, p=J$, $m=l-1$ and $n=l+1$.

To establish $J$ decoupled error sensor signals, a projection matrix $\mathcal{S}_{l}^{\perp} \in \mathbb{C}^{M \times M}$ is computed:

$$
\mathcal{S}_{l}^{\perp}=\left\{\mathbf{I}-\mathcal{S}_{l}\left[\mathcal{S}_{l}^{H} \mathcal{S}_{l}\right]^{-1} \mathcal{S}_{l}^{H}\right\} .
$$

Proposition 3.1. $\mathcal{S}_{l}^{\perp}$ defined in equation (10) results in a non-zero solution if $M>K(J-1)$, assuming $\mathcal{S}_{l}^{H} \mathcal{S}_{l}$ is full rank. Furthermore, for the $M R-P C L M S$ algorithm to be feasible (later) it follows that:

$$
M \geq K J .
$$

Proof. The proof is omitted for brevity, but can be derived from linear algebra theory [3].

Note that inequality (11) poses a constraint on the number of error sensors $M$. The constraint is mild though, since commonly $M>>K$. Using the projection matrix $\mathcal{S}_{l}^{\perp}, \widetilde{\mathbf{e}}(k)$ in equation (7) can be pre-multiplied with $\mathcal{S}_{l}^{\perp}$, to create:

$\mathcal{S}_{l}^{\perp} \widetilde{\mathbf{e}}(k)=\mathcal{S}_{l}^{\perp} \widetilde{\mathbf{S}}_{l}\left(\widetilde{\mathbf{W}}_{l}^{o}+\widetilde{\mathbf{W}}_{l}(k)\right)+\mathcal{S}_{l}^{\perp} \mathcal{S}_{l} \mathcal{W}_{\mathbf{l}}(k)$.

Since $\mathcal{S}_{l}^{\perp} \mathcal{S}_{l}=\mathbf{0}$, equation (12) reduces to:

$$
\widetilde{\mathbf{e}}_{l}(k)=\mathcal{S}_{l}^{\perp} \widetilde{\mathbf{e}}(k)=\mathcal{S}_{l}^{\perp} \widetilde{\mathbf{S}}_{l}\left(\widetilde{\mathbf{W}}_{l}^{o}+\widetilde{\mathbf{W}}_{l}(k)\right) .
$$

Equation (13) can be applied for every $l=1, \ldots, J$ to establish $J$ decoupled filtered error sensor signals $\widetilde{\mathbf{e}}_{l}(k),(l=1, \ldots, J)$.

Proposition 3.2. Given $\mathcal{S}_{l}$ as defined in equation (8), and $\widetilde{\mathbf{S}}_{l}$ as in equation (7), both $\mathcal{S}_{l}^{\perp}$ and $\mathcal{S}_{l}^{\perp} \widetilde{\mathbf{S}}_{l}$ can be computed numerically robust by using the following QR factorization [3]:

$$
\left[\begin{array}{ll}
\mathcal{S}_{l} & \widetilde{\mathbf{S}}_{l}
\end{array}\right]=\left[\begin{array}{ll}
\mathbf{Q}_{1_{l}} & \mathbf{Q}_{2_{l}}
\end{array}\right]\left[\begin{array}{cc}
\mathbf{R}_{11_{l}} & \mathbf{R}_{12_{l}} \\
\mathbf{0} & \mathbf{R}_{22_{l}}
\end{array}\right]
$$

and is given by:

$$
\mathcal{S}_{l}^{\perp}=\mathbf{I}-\mathbf{Q}_{1_{l}} \mathbf{Q}_{1_{l}}^{H}, \quad \mathcal{S}_{l}^{\perp} \widetilde{\mathbf{S}}_{l}=\mathbf{Q}_{2_{l}} \mathbf{R}_{22_{l}},
$$

with $\mathbf{Q}_{1_{l}} \in \mathbb{C}^{M \times K(J-1)}, \mathbf{Q}_{2_{l}} \in \mathbb{C}^{M \times M-K(J-1)}$, $\mathbf{R}_{11_{l}} \in \mathbb{C}^{K(J-1) \times K(J-1)}, \mathbf{R}_{12_{l}} \in \mathbb{C}^{K(J-1) \times K}$ and $\mathbf{R}_{22_{l}} \in \mathbb{C}^{M-K(J-1) \times K}$.
Proof. The proof is omitted for brevity, but can be derived by using theory from [4].

For the derivation of the MR-PCLMS algorithm we proceed along similar lines as for the PCLMS algorithm. A SVD can be computed of $\mathcal{S}_{l}^{\perp} \widetilde{\mathbf{S}}_{l}$ :

$$
\mathcal{S}_{l}^{\perp} \widetilde{\mathbf{S}}_{l}=\mathbf{Q}_{2_{l}} \mathbf{R}_{22_{l}}=\mathbf{U}_{l} \boldsymbol{\Sigma}_{l} \mathbf{V}_{l}^{H} .
$$

Using the outcome of the SVD in equation (14), equation (13) can be pre-multiplied by $\mathbf{U}_{l}^{H}$ :

$$
\overline{\widetilde{\mathbf{e}}}_{l}(k)=\mathbf{U}_{l}^{H} \widetilde{\mathbf{e}}_{l}(k)=\overline{\widetilde{\mathbf{d}}}_{l}+\boldsymbol{\Sigma}_{l} \overline{\widetilde{\mathbf{W}}}_{l}(k),
$$

where $\overline{\widetilde{\mathbf{d}}}_{l}=\mathbf{U}_{l}^{H} \mathcal{S}_{l}^{\perp} \widetilde{\mathbf{S}}_{l} \widetilde{\mathbf{W}}_{l}^{o}$ and with $\overline{\widetilde{\mathbf{W}}}_{l}(k)=$ $\mathbf{V}_{l}^{H} \widetilde{\mathbf{W}}_{l}(k)$. With equation (15) a form equal to equation (4) has been established. Now $J$ parallel least mean squares algorithms can be used to update $J$ decoupled adaptive controllers by minimizing $J_{l}(k)=\overline{\mathbf{e}}_{l}^{T}(k) \overline{\mathbf{e}}_{l}(k)$ with respect to $\overline{\mathbf{w}}_{l}(k)$ :

$$
\overline{\mathbf{w}}_{l}(k+1)=\overline{\mathbf{w}}_{l}(k)-\frac{\mu}{2} \frac{\partial \overline{\mathbf{e}}_{l}^{T}(k) \overline{\mathbf{e}}_{l}(k)}{\partial \overline{\mathbf{w}}_{l}(k)}
$$

for $l=1, \ldots, J$. In figure 4 the MR-PCLMS adaptive control problem is depicted. In the figure the argument $q^{-1}$ has been dropped from the filters. Note that in the actual implementation, the matrices $\mathbf{U}_{l}^{H}$ and $\mathcal{S}_{l}^{\perp},(l=1, \ldots, J)$, can be multiplied first to obtain the matrix $\boldsymbol{\Phi}_{l}=\mathbf{U}_{l}^{H} \mathcal{S}_{l}^{\perp}=$ $\mathbf{U}_{l}^{H}\left(\mathbf{I}-\mathbf{Q}_{1_{l}} \mathbf{Q}_{1_{l}}^{H}\right)$. $\boldsymbol{\Phi}_{l}$ can then be transformed to the time-domain to obtain $\boldsymbol{\Phi}_{l}\left(q^{-1}\right)$, which can be implemented (note that $\boldsymbol{\Phi}_{l}\left(q^{-1}\right)$ must be designed with care). This way the parameters which need to be implemented are reduced, which decreases the processor load. Remark: To circumvent the constraint in equation (11), another way to establish $J$ decoupled error sensor signals $\overline{\mathbf{e}}_{l}(k)$, $(l=1, \ldots, J)$, is to design $J$ frequency selective filters $\mathcal{F}_{l}\left(q^{-1}\right)$, where every $\mathcal{F}_{l}\left(q^{-1}\right)$ only passes the particular frequency at normalized frequency $\omega_{l} T$ (i.e. $\left.\mathbf{e}_{l}(k)=\mathcal{F}_{l}\left(q^{-1}\right) \mathbf{e}(k)\right)$. We do not elaborate on that further.

\section{SIMULATION RESULTS}

The MR-PCLMS algorithm was tested on a model of a vibration isolation set-up. The model consists of three reference inputs $(J=3)$, two actuator inputs $(K=2)$ and six error sensor outputs $(M=6)$. Thus satisfying the inequality in equation (11). $\mathbf{S}\left(q^{-1}\right)$ was established from real-time data by subspace identification between two actuator inputs and six error sensor outputs of a sixdegrees-of-freedom vibration isolation set-up [5]. For simplicity, the optimal controller $\mathbf{W}_{\text {opt }}\left(q^{-1}\right)$ 
was selected as a FIR controller with twelve optimal parameters. $\mathbf{P}\left(q^{-1}\right)$ was chosen as $\mathbf{P}\left(q^{-1}\right)=$ $-\mathbf{S}\left(q^{-1}\right) \mathbf{W}_{\text {opt }}\left(q^{-1}\right)$. The sample frequency is 3 kHz. Every reference input $x_{l}(k),(l=1,2,3)$, contains a single frequency component. Here we choose three natural frequencies of $\mathbf{S}\left(q^{-1}\right)$ i.e. $f_{1}=$ $348 \mathrm{~Hz}, f_{2}=410 \mathrm{~Hz}$ and $f_{3}=480 \mathrm{~Hz}$. We assume perfect knowledge of $\mathbf{S}\left(q^{-1}\right)$. Moreover, we put uncorrelated stochastic white noise on $\mathbf{d}(k)$ with a signal to noise ratio of $30 \mathrm{~dB}$. The step-sizes $\mu_{l}$ in equation (16) were tuned to be 0.05 . The results are averaged over 100 independent runs. In figure 3 the normalized (to $0 \mathrm{~dB}$ ) ensemble average learning curve is given averaged over $\mathbf{e}(k)$. Af-

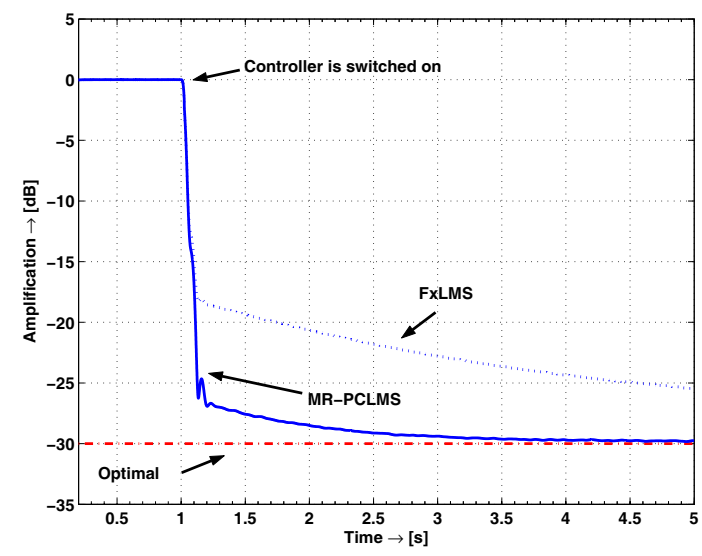

Fig. 3. Averaged ensemble average learning curves.

ter five seconds, a reduction of $29.7 \mathrm{~dB}$ is accomplished in $\mathbf{e}(k)$. The reduction is not optimal (i.e. $30 \mathrm{~dB}$ ) due to a minor misadjustment [1]. The MRPCLMS algorithm outperforms the FxLMS algorithm which converges slower and obtains $25.4 \mathrm{~dB}$ reduction (with $\mu=0.05$ ). Note that the curves have been filtered for better view.

\section{CONCLUSION}

In this paper a multiple reference extension was proposed to the principle component least mean squares (PCLMS) algorithm. The new algorithm enables the PCLMS algorithm with the ability to suppress a disturbance signal which contains multiple frequency components. A restriction however is that the number of error sensor outputs $M$, the number of actuator inputs $K$ and the number of reference inputs $J$, must satisfy $M \geq K J$. Simulation experiments on a realistic model of a vibration isolation set-up demonstrated the potential of the algorithm. Finally, the authors want to thank master's thesis student F. Niewold for the filtered$\mathrm{x}$ least mean square simulation experiments with the Active Control Toolbox for Matlab.

\section{REFERENCES}

[1] Stephen Elliott, Signal Processing for Active Control, Academic Press, 2000.

[2] Randolph H. Cabell, A Principal Component Algorithm for Feedforward Active Noise and Vibration Control, Ph.D. thesis, Virginia Polytechnic Institute and State University, Blacksburg, Virginia, USA, 1998.

[3] Gene H. Golub and Charles F. Van Loan, Matrix computations, The John Hopkins University Press, 1996.

[4] L.R.J. Haverkamp, State space identification, Ph.D. thesis, Delft University of Technology, Delft, The Netherlands, 2001.

[5] G. Nijsse, H. Super, J. Van Dijk, and J.B. Jonker, "Subspace based identification and control of a six-degrees-of-freedom experimental vibration isolation set-up," in Fifth European conference on noise control engineering, Napels, Italy, May 2003.

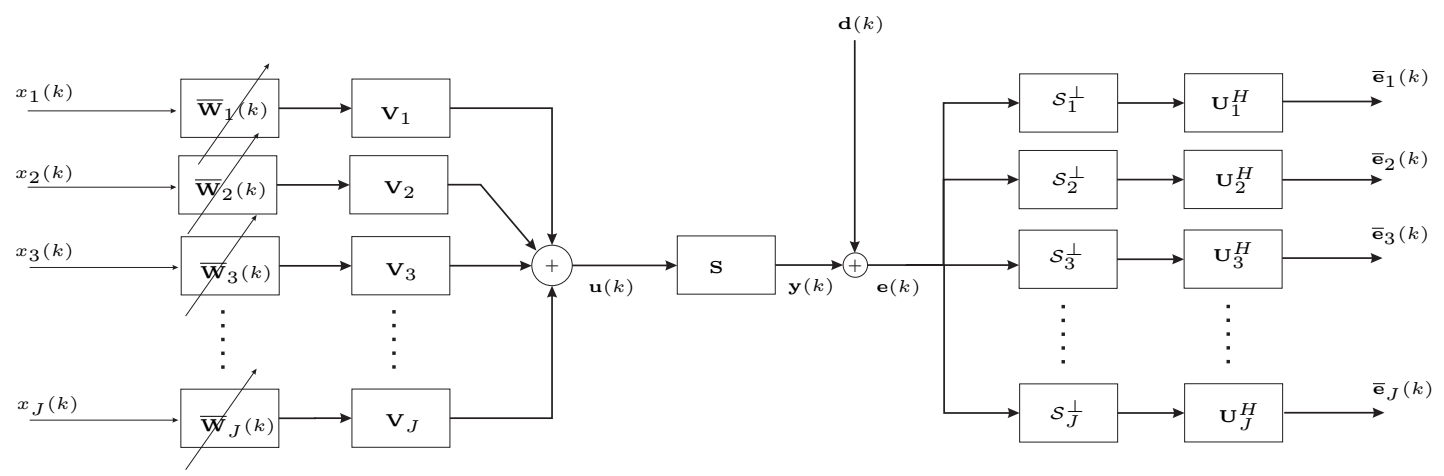

Fig. 4. MR-PCLMS adaptive control problem. 